\title{
Fabrication of superconducting tantalum nitride thin films using infra-red pulsed laser deposition
}

\author{
S. Chaudhuri, $1, *$ L. Chandernagor, ${ }^{1,2}$ M. Lahtinen, ${ }^{3}$ M. Ging, ${ }^{1,2}$ and I. J. Maasilta ${ }^{1}$ \\ ${ }^{1}$ Nanoscience Center, Department of Physics, University of Jyväskylä, \\ P. O. Box 35, FIN-40014 University of Jyväskylä, Finland \\ ${ }^{2}$ ENSICAEN, 6 bd Maréchal Juin, F-14050 CAEN Cedex 4 France \\ ${ }^{3}$ Department of Chemistry, Laboratory of Inorganic and Analytical Chemistry, \\ P.O. Box 35, FIN-40014 University of Jyväskylä, Finland
}

(Dated: October 17, 2018)

\begin{abstract}
We report the successful fabrication of superconducting tantalum nitride (TaN) thin films using a pulsed laser deposition technique with $1064 \mathrm{~nm}$ radiation. Films with thickness $\sim 100 \mathrm{~nm}$ deposited on $\mathrm{MgO}(100)$ single crystals and on oxidized silicon $\left(\mathrm{SiO}_{2}\right)$ substrates exhibited a superconducting transition temperature of $\sim 8 \mathrm{~K}$ and $6 \mathrm{~K}$, respectively. The topography of these films were investigated using atomic force and scanning electron microscopy, revealing fairly large area particulate free and smooth surfaces, while the structure of the films were investigated using standard $\theta-2 \theta$ and glancing angle X-ray diffraction techniques. For films grown on $\mathrm{MgO}$ a face-centered cubic phase of TaN was observed, while films grown on $\mathrm{SiO}_{2}$ exhibited the face-centered cubic and as well as a mononitride hexagonal phase. The transition temperature of the TaN deposited on $\mathrm{SiO}_{2}$ was found to be more sensitive to the nitrogen pressure during deposition as compared to the TaN deposited on $\mathrm{MgO}$.
\end{abstract}

\section{INTRODUCTION}

Efficient fabrication of materials with intermediate and high superconducting transition temperature $\left(T_{C}\right)$ is essential for the development and wide-spread use of superconducting electronics. Particularly attractive are those materials whose $T_{C}$ is above $4.2 \mathrm{~K}$, the boiling point of liquid $\mathrm{He}^{4}$. There exists a host of materials fulfilling this criteria ranging from elementary metals like $\mathrm{Nb}\left(T_{C} \sim\right.$ $9 \mathrm{~K}$ ) to more complex multi-element based copper oxide materials $\left(T_{C} \text { up to } \sim 135 \mathrm{~K}\right)^{\underline{1}}$. However, the superconducting properties of such multi-element materials are extremely sensitive to stoichiometry, and thus their fabrication is non-trivial. A good compromise in terms of $T_{C}$ and ease of fabrication are metal nitrides as they are binary in composition but their $T_{C}$ s are higher than their elemental counterparts. Particularly, the nitride of tantalum $(\mathrm{TaN})$ in thin film form is a promising material for micro-electronic device applications. In its normal state, TaN has earned the reputation as an excellent diffusion barrier material ${ }^{2.3}$, as resistors with low temperature coefficient $\underline{4}^{\underline{4}}$ and as hard coatings ${ }^{\underline{5}}$, while superconducting TaN was recently demonstrated to be a much better candidate than $\mathrm{NbN}$ for single photon detection ${ }^{6}$ because of its lower gap and lower density of states. TaN also works well as a normal state barrier in SNS Josephson junctions $\frac{7.8}{}$.

Depending on the amount of incorporated nitrogen, $x$, the tantalum nitride system $\mathrm{TaN}_{x}$ can exhibit a variety of crystallographic phases like cubic, hexagonal or tetragonal ${ }^{9.10}$. Among these, the hexagonal and tetragonal phases are thermodynamically stable 11 , but with no signature of superconductivity down to $1.5 \mathrm{~K}^{12,13}$. The stoichiometric mononitride (TaN) phase possess a face-centered cubic (FCC) structure and exhibits superconductivity with a $T_{C}$ of $8.15 \mathrm{~K}^{14}$, which under spe- cial preparation conditions, has been pushed up to 10.8 $\mathrm{K}^{12}$. Thermodynamically this phase is stable only at high temperatures $\sim 1800{ }^{\circ} \mathrm{C}$ or under high pressures 15 , but it is metastable at room temperature. Nevertheless, the metastable FCC phase can be obtained at low temperatures for TaN thin films prepared by plasma assisted or ion irradiated process, whereby the energetic ions provides the necessary energy of crystallization for the FCC phase formation $\underline{16}$. $\mathrm{TaN}_{x}$ thin films have been fabricated mainly by reactive sputtering $3,6,10,14,17-21$ and nitrogen implantation of evaporated tantalum ${ }^{5,9,16,22}$ on a variety of substrates. In most cases the fabricated films were intended for normal state applications and therefore the superconducting aspects were not investigated. So far, superconducting TaN thin films have been prepared on sapphire $e^{\frac{6}{}}$, glass ${ }^{14}$, quartz ${ }^{17}$ and alumina ${ }^{14}$ substrates, all using reactive sputtering. TaN thin films (with no reported superconductivity) have also been fabricated using pulsed laser deposition (PLD) technique where the first harmonic (532 $\mathrm{nm}$ radiation) from a Nd:YAG laser was used as the source 23,24 . The first harmonic has been traditionally used with Nd:YAG lasers in PLD, however it has the disadvantage that the output power is much reduced compared to the fundamental $1064 \mathrm{~nm}$ radiation. We have recently shown that it is actually possible to obtain high quality superconducting $\mathrm{NbN}$ films using just the fundamental $1064 \mathrm{~nm}$ radiation from a Nd:YAG laser ${ }^{25}$, and the same tactic was used in this work for TaN deposition.

In this paper, we report the successful fabrication of superconducting TaN thin films on (100) oriented single crystals of $\mathrm{MgO}$ and oxidized (100) silicon $\left(\mathrm{SiO}_{2}\right)$ substrates. The films were fabricated by the ablation of a high purity Ta target in a ultra high purity nitrogen environment using the fundamental $1064 \mathrm{~nm}$ laser pulse emission from a Nd:YAG laser. We have tried to 
maximize the $T_{C}$ by carefully optimizing the growth parameters. Since our long term goal is the fabrication of superconducting TaN based tunnel junction devices, in the present study we have investigated the topographical, structural and the electrical aspects of our thin films. Our recent results on $\mathrm{NbN}^{26}$ and $\mathrm{Nb}^{27}$ based tunnel junction micro-devices hint that intermediate $T_{C}$ based thin film superconductors, such as TaN, may be the key to the practical realization of high operation temperature $(1 \mathrm{~K})$ electronic coolers ${ }^{28,29}$.

\section{EXPERIMENTAL}

TaN thin film of thickness $\sim 100 \mathrm{~nm}$ were fabricated by ablating a high purity $(99.9 \%)$ Ta target in an ultra high purity nitrogen environment. For ablation, $4 \mathrm{~ns}$ laser pulses corresponding to the fundamental $(\lambda=1064$ $\mathrm{nm}, h \nu=1.16 \mathrm{eV})$ emission of an Nd:YAG laser were used. The details of the $1064 \mathrm{~nm}$ radiation based PLD technique can be found elsewhere ${ }^{25}$. The laser was operated at a frequency of $10 \mathrm{~Hz}$, the substrate to target distance was $5 \mathrm{~cm}$ while the incident energy density was $\sim 6 \mathrm{Jcm}^{-2}$. Typical growth rate was $\sim 4 \mathrm{~nm} / \mathrm{s}$. TaN films were deposited on two types of substrates : (100) oriented single crystals of $\mathrm{MgO}$ (purchased from Crystec $\mathrm{GmbH}$ ) and thermally oxidized (100) silicon substrates (oxide thickness $\sim 300 \mathrm{~nm}$ ). Since for any potential device application the leakage current to the substrate has to be minimized, oxidized silicon was chosen over bare silicon due to the excellent dielectric properties of silicon oxide thin films. The deposition temperature $\left(T_{D}\right)$ was kept fixed at $700{ }^{\circ} \mathrm{C}$, as previous studies suggested that higher $T_{D}$ favours the formation of larger grains ${ }^{23}$. The nitrogen pressure $\left(p_{N}\right)$ was varied to change the Ta:N ratio in the films. After deposition, the films were let to cool back to room temperature in a constant flow of high purity nitrogen at the same pressure as at which the TaN was deposited. Also, films corresponding to the same pressure but different substrates were fabricated during the same deposition process. Room temperature atomic force microscopy (AFM) was carried out in the tapping mode using a Nanoscope V AFM. The X-ray diffraction analyses on the films were made with PANalytical X'Pert Pro alpha 1 diffractometer using glancing angle of $5^{\circ}$ and Johansson monochromatized $\mathrm{Cu} \mathrm{K} \mathrm{K}_{\alpha 1}$ radiation $(\lambda=1.5406 \AA$; tube settings: $45 \mathrm{kV}, 30 \mathrm{~mA})$. For each measurement, the sample plate was prepared on a zero-background holder made from silicon. The data was collected from a spinning sample by X'Celerator detector in the $2 \theta$-range of $10-110^{\circ}$ with a step size of $0.017^{\circ}$ and counting times of $1000 \mathrm{~s}$ (480 s for glancing angle) per step. The diffraction data was handled by X'Pert HighScore Plus v. 2.2d software package and the ICDD PDF $4+$ powder diffraction database was used for the qualitative search-match phase identification. Resistivity measurements were carried out in a four probe configuration using a dipstick immersed in a liquid helium bath, with a current bias of $10 \mu \mathrm{A}$.

\section{RESULTS}

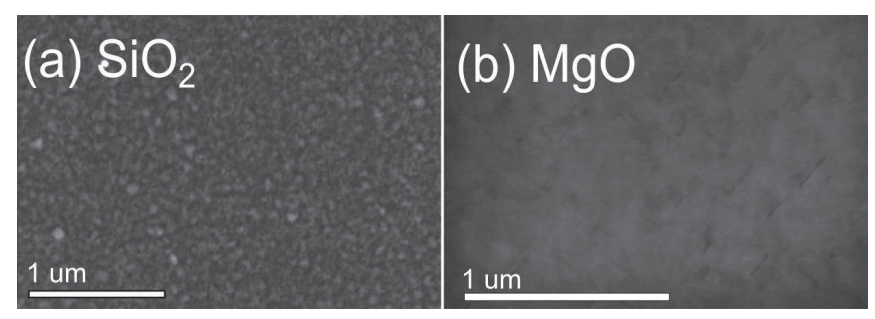

FIG. 1. Room temperature scanning electron micrograph of TaN grown on (a) $\mathrm{SiO}_{2}$ and (b) $\mathrm{MgO}$ respectively. The length scale is indicated by the horizontal white line on bottom left.

Scanning electron microscopy images on TaN grown on both $\mathrm{SiO}_{2}$ and $\mathrm{MgO}$ reveal a very low particulate density, as seen from Fig. 11 It is easy to obtain particulate free regions that are up to several tens of microns in dimension. The grainy texture of the TaN film on $\mathrm{SiO}_{2}$ is clearly visible, while the film on $\mathrm{MgO}$ appears much more uniform.

The surface topography of the TaN films were investigated using atomic force microscopy, with the results for $5 \times 5 \mu \mathrm{m}^{2}$ scans shown in Fig. 2. The root mean square surface roughness for the films grown on $\mathrm{MgO}$ and $\mathrm{SiO}_{2}$ were 2.2 and $2 \mathrm{~nm}$, respectively, with the line scans showing the roughness of the surface measured along one of the diagonals of the scan area are shown in Fig. 2(c). It is clearly seen that the morphologies of the films grown on the two different substrates are quite different from each other. The spatial scale of the surface roughness of the film grown on $\mathrm{SiO}_{2}$ is much smaller than the corresponding one in the film grown on $\mathrm{MgO}$. These TaN films tend to be smoother than NbN films of similar thickness prepared in the same PLD chamber, which revealed an rms surface roughness of $\sim 3 \mathrm{~nm}^{25}$. Using the rougher $\mathrm{NbN}$ films, we have already successfully fabricated $\mathrm{NbN}$ based tunnel junction micro-thermometers ${ }^{26}$ capable of operating from $0.1 \mathrm{~K}$ upto $T_{C}$ of $\mathrm{NbN}^{26}$. Thus, the low particulate density in conjunction with the intrinsic smoothness of the surface is very promising for the fabrication of TaN based micro scale tunnel junction devices.

Structural analysis of the TaN thin films, carried out at room temperature using the traditional $\theta-2 \theta$ scanning method, revealed diffraction peaks originating both from the substrate and the TaN film. As seen from Fig. 3 (a) and (b), the diffractograms are dominated by the respective substrate peaks. Results of glancing angle measurements, where only the peaks from the films are observed, are displayed in Fig. 3 (c) and (d). Analyzing both the $\theta-2 \theta$ and the glancing angle data, we find that TaN films grown on the $\mathrm{SiO}_{2}$ substrate exhibit distinct peaks corresponding to (111), (200), (220), (311) and (222) planes of a face centered cubic (FCC) structure. The simultaneous 


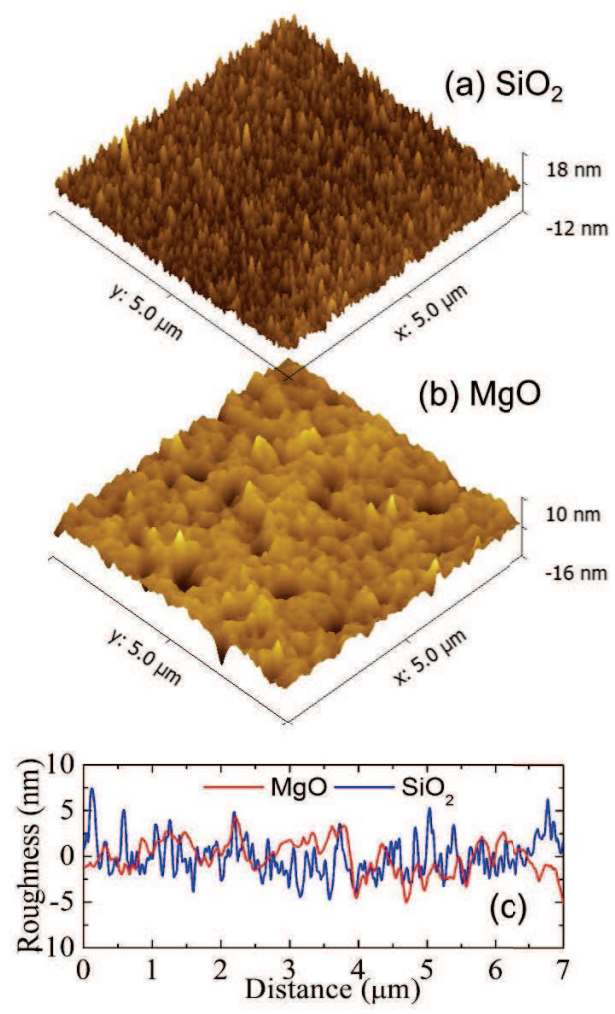

FIG. 2. [Color online] Room temeperature atomic force microscopy images of TaN thin film grown on (a) $\mathrm{SiO}_{2}$ and (b) $\mathrm{MgO}$ substrates deposited at the same time at $p_{N}=55.5$ mTorr. (c) Line scans repersenting the variation in surface roughness measured along one of the diagonals of the scanned area.

occurrence of reflections from all these planes indicates that our TaN film on $\mathrm{SiO}_{2}$ consists of FCC TaN grains with random orientations. TaN thin films grown on silicon and glass substrates at low temperatures have also been shown to exhibit such a poly-crystalline nature ${ }^{10,16}$. However, the films grown on the $\mathrm{MgO}$ substrate exhibit a dominant (200) reflection indicating that there is a preferred growth direction. It has been observed that when TaN was deposited using metal evaporation under simultaneous nitrogen ion irradiation, the preferred growth direction changes from (111) to (100) with increasing irradiation intensity $\underline{\underline{16}}$.

Using the Bragg formula, $2 d \sin \theta=n \lambda$, we obtain a value for the out-of-plane lattice parameter, $c \sim 4.4 \AA$ for the FCC phase of the TaN films grown on both $\mathrm{MgO}$ and $\mathrm{SiO}_{2}$. In addition to the peaks associated with the FCC phase, the diffractograms of TaN grown on $\mathrm{SiO}_{2}$ exhibit other peaks that possibly correspond to reflections from (001) and (300) planes of the hexagonal TaN phase, which has also been observed for TaN grown on $(111)^{30}$ and $(100)^{23}$ Si substrates at a high temperature and substrate bias, respectively. In our case, the peak occurring at $2 \theta \sim 30.6^{\circ}$ corresponds to an out-of-plane lattice parameter $c=2.92 \AA$, close to what is expected for hexagonal $\epsilon$-TaN $(c=2.91 \AA)^{\underline{9}}$. Similar to FCC TaN,
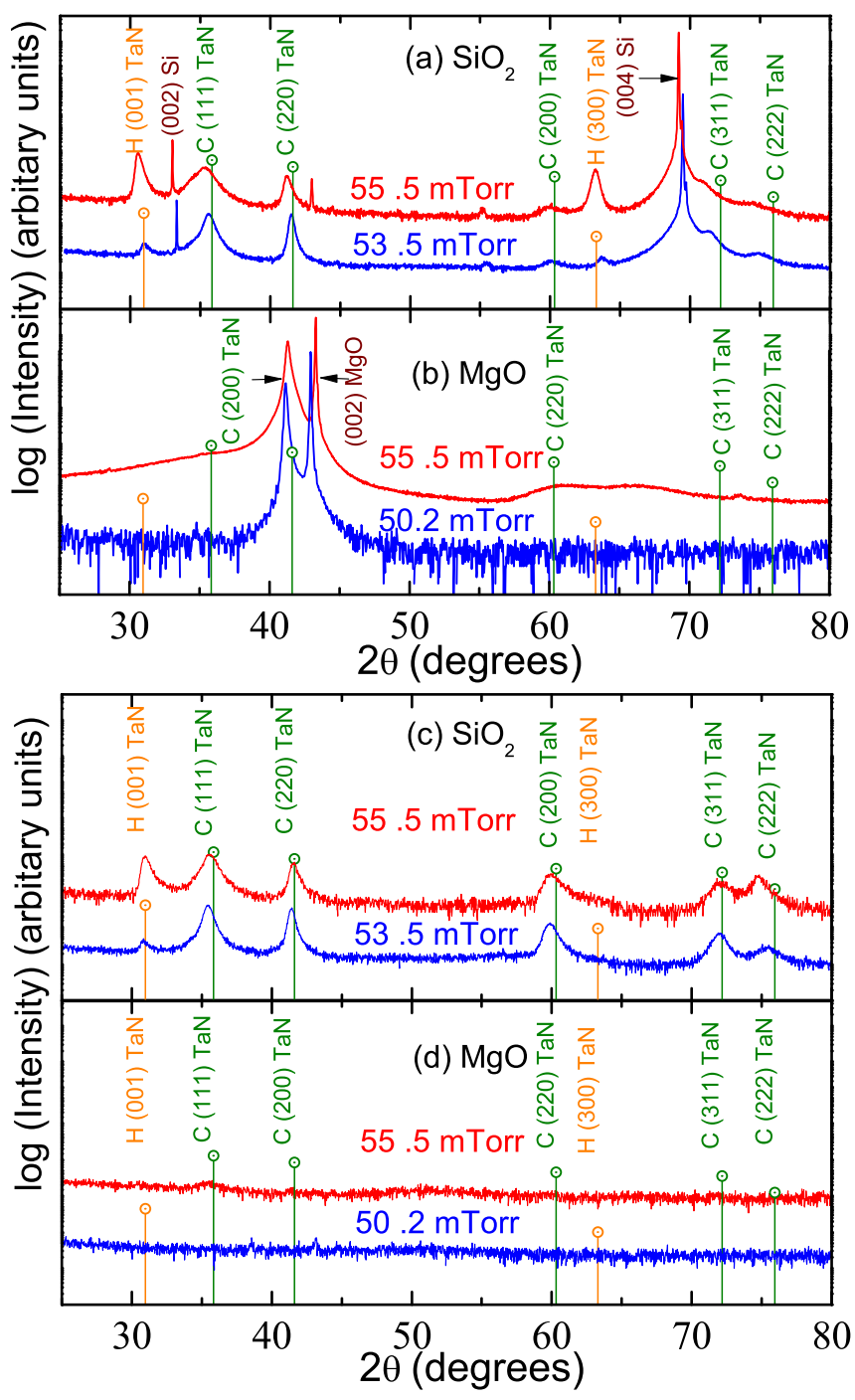

FIG. 3. [Color online] Room temperature X-ray diffraction patterns of TaN films deposited on (a) oxidized (100) Si and (b) (100) $\mathrm{MgO}$ substrates measured in $\theta-2 \theta$ mode. In each case diffractograms corresponding to $p_{N}=55.5$ (red) and 53.5 (blue) mTorr on $\mathrm{SiO}_{2}$ and 55.5 (red) and 50.2 (blue) mTorr on $\mathrm{MgO}$ is shown. The corresponding glancing angle measurements are shown in panels (c) and (d). The reflections from various lattice planes of cubic $(C)$ and hexagonal $(H)$ phases have been identified and marked, with the characteristic positions of the cubic and hexagonal TaN phases indicated by the green and orange tick marks, respectively.

this hexagonal phase is also stoichiometrically mononitride, thus the identical stoichiometry is expected to lead to the co-existence of the two phases. The peak occurring at $2 \theta \sim 63.2^{\circ}$ corresponds to an in-plane lattice parameter $a \sim 5.07 \AA$, close to the expected value of $5.19 \AA$ for the hexagonal $\epsilon$-TaN ${ }^{9}$. Thus, just as the FCC phase, the hexagonal phase is polycrystalline, as well. It can also be seen from Fig. 3(a) and (c) that the peak intensities of the hexagonal TaN phase diminish with decreasing $p_{N}$.

For both the FCC and hexagonal structures the TaN 


\begin{tabular}{ccccccc}
\hline \hline Peak position & Miller & Crystal & \multicolumn{5}{c}{ Crystallite size $(\AA)$} \\
$(2 \theta)$ & indices & structure & $\mathrm{SiO}_{2}$ (mTorr) & $\mathrm{MgO}$ (mTorr) \\
(degrees) & & & 55.5 & 53.5 & 55.5 & 50.2 \\
\hline 35.58 & 111 & Cubic & 71 & 121 & & \\
41.57 & 200 & Cubic & 165 & 224 & 453 & 817 \\
60.4 & 220 & Cubic & 84 & 83 & & \\
72.06 & 311 & Cubic & 90 & 90 & & \\
89.23 & 400 & Cubic & 146 & 135 & 206 & 352 \\
30.74 & 001 & Hexagonal & 185 & 157 & & \\
63.24 & 300 & Hexagonal 196 & 181 & & \\
& & & & & & \\
\hline \hline
\end{tabular}

TABLE I. Crystallite sizes associated with various orientations of TaN grains grown on $\mathrm{SiO}_{2}$ and on $\mathrm{MgO}$ substrates extracted from our X-ray data.

peaks are broad, indicating a small crystallite size $w$. Using the Debey-Scherrer equation, $w=0.9 \lambda /(\beta \cos \theta)$, where $\beta$ is the FWHM of a single diffraction peak, we estimated the size of the crystallites, as listed in Table [ For films grown on $\mathrm{SiO}_{2}$ at $53.5 \mathrm{~m}$ Torr and on $\mathrm{MgO}$ at 50.2 mTorr, the peaks corresponding to the FCC TaN phase are slightly narrower than the corresponding films grown at 55.5 mTorr, indicating a larger crystallite size. For example on $\mathrm{SiO} 2$, the crystallite sizes vary from 83 to $224 \AA$ for $p_{N}=53.5 \mathrm{mTorr}$, in contrast to $\sim$ 71 to $165 \AA$ for $p_{N}=55.5$ mTorr. Similarly, the FCC crystallite size of $\mathrm{TaN}$ grown on $\mathrm{MgO}$ varies from 206 $453 \AA$ for $p_{N}=50.2 \mathrm{mTorr}$, to $352-817 \AA$ for $p_{N}=$ 55.5 mTorr. In all cases, the (200) oriented crystallites possess the largest size, ranging from $\sim 453-817 \AA$ on $\mathrm{MgO}$ to $165-244 \AA$ on $\mathrm{SiO}_{2}$. Interestingly, in most cases $w$ is larger than the observed coherence length in some TaN thin films $\sim 90 \AA 31$.

In case of the $\mathrm{MgO}$ samples, the extremely broad cubic TaN peaks (111) and (220) disappear as the pressure decreases from 55.5 to 50.2 mTorr, as seen in Fig. 3 (b). Furthermore, the clear absence of the FCC peaks in the glancing angle data of $\mathrm{TaN}$ on $\mathrm{MgO}$ indicates that the very surface of the films grown on $\mathrm{MgO}$ is amorphous, and the crystalline phases exists only closer to the substrate-film interface. These peaks start to be visibile only when the glancing angle is increased to $8^{\circ}$. Similarly, the hexagonal (300) peak of $\mathrm{TaN}$ on $\mathrm{SiO}_{2}$ is also absent in the surface region. The fact that the surface of the TaN film on $\mathrm{MgO}$ is amorphous, and that the hexagonal and the cubic phases differ in grain size and morphology $\underline{\underline{10}}$ are possibly the reasons behind the different observed morphologies of the films grown on $\mathrm{MgO}$ and $\mathrm{SiO}_{2}$ in our AFM measurements.

The variation of the normalized resistivity of two TaN

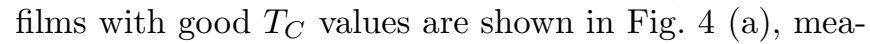
sured from room temperature down to liquid helium temperature $4.2 \mathrm{~K}$. The film on $\mathrm{MgO}$ was grown at $p_{N}=55.5$ mTorr, whereas for the film on $\mathrm{SiO}_{2}, p_{N}=53.5$ mTorr. The room temperature resistivity value were $\sim 50$ and $150 \mu \Omega \mathrm{cm}$ on the $\mathrm{MgO}$ and $\mathrm{SiO}_{2}$, respectively. We see that upon cooling from room temperature, both films
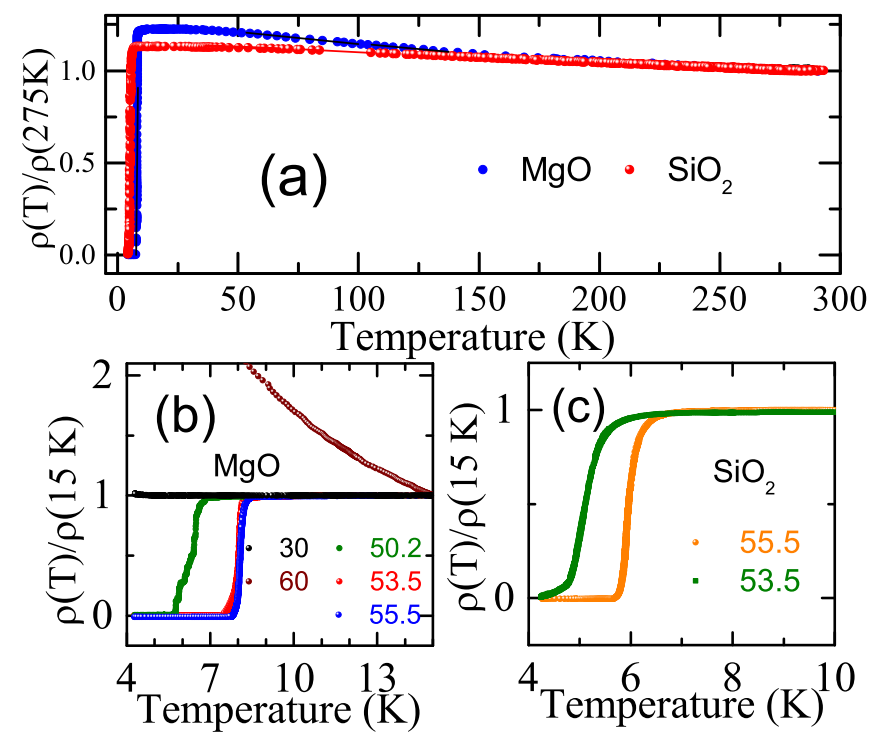

FIG. 4. [Color online] (a) Temperature dependence of the resistivity normalized to the values at $275 \mathrm{~K}$, of TaN film grown at $700^{\circ} \mathrm{C}$, revealing a superconducting transition of $\sim 8 \mathrm{~K}$ and $5 \mathrm{~K}$ on $\mathrm{MgO}$ and $\mathrm{SiO}_{2}$ substrates, respectively. The respective $p_{N}$ were 55.5 and 53.5 mTorr. Temperature dependence of the resistivity of a TaN film, normalized to its value at 15 $\mathrm{K}$, grown on (b) $\mathrm{MgO}$ and (c) $\mathrm{SiO}_{2}$ at several values of $p_{N}$ indicated. Th highest obtained $T_{C}$ were $8 \mathrm{~K}$ and $6 \mathrm{~K}$ on $\mathrm{MgO}$ and $\mathrm{SiO}_{2}$, respectively.

exhibited a negative temperature coefficient behaviour all the way down till $T_{C}$. The residual resistivity ratio $\rho(275 K) / \rho(20 K)$ was $\sim 0.7-0.8$. In Fig. 田(b) and (c) we show the resistive behaviour of films grown on $\mathrm{MgO}$ and $\mathrm{SiO}_{2}$, respectively, at various $p_{N}$. For films grown on $\mathrm{MgO}$, at $p_{N}=30 \mathrm{mTorr}$, the resistivity remained almost constant with no signature of $T_{C}$ down to $4.2 \mathrm{~K}$. Upon increasing $p_{N}$ to 50.2 mTorr, a superconducting transition occurs at $T_{C} \sim 6 \mathrm{~K}$, which can be pushed up to $\sim 8 \mathrm{~K}$ when $p_{N}=53.5-55.5$ mTorr. At $p_{N}=60$ mTorr, an insulating phase is observed with no signature of $T_{C}$ down to $4.2 \mathrm{~K}$. On the $\mathrm{SiO}_{2}$ substrate, superconductivity was observed only in films grown at $p_{N}=53.5-55.5$ mTorr. So far, the highest values of $T_{C}$ obtained on $\mathrm{MgO}$ and $\mathrm{SiO}_{2}$ were grown at $p_{N}=55.5 \mathrm{mTorr}$, giving $T_{C}$ values of $\sim 8 \mathrm{~K}$ and $6 \mathrm{~K}$ for $\mathrm{MgO}$ and $\mathrm{SiO}_{2}$ substrates, respectively. The corresponding widths of the transitions were about 0.25 and $0.4 \mathrm{~K}$, respectively.

Although the lattice constants of the FCC phases of $\mathrm{TaN}$ on $\mathrm{MgO}$ and $\mathrm{SiO}_{2}$ are identical, their $T_{C}$ values differ significantly. We speculate that this is possibly because on $\mathrm{SiO}_{2}$, due to the exsistence of the two different phases (superconducting FCC and non-superconducting hexagonal), the effective thickness of the superconducting FCC phase is less than in the $\mathrm{MgO}$ counterpart. It is well know that the $T_{C}$ of thin films is thickness dependent, whereby $T_{C}$ typically decreases with decreasing film thickness 32 . On the other hand, a comparison of the films grown on $\mathrm{SiO}_{2}$ at 53.5 and 55.5 mTorr (Fig. 4 (c)) 
shows that although the hexagonal phase fraction is comparatively lower and FCC grain size is larger in the film grown at 53.5 mTorr (Fig. 3(c)), its $T_{C}$ is about $1 \mathrm{~K}$ lower than the for the film grown at $p_{N}=55.5$ mTorr. A similar situation also arises for films grown on $\mathrm{MgO}$. In this case, the film grown at $p_{N}=50.2$ mTorr possess a larger FCC grain size and a reduced $T_{C}$ as compared to film grown at $p_{N}=55.5 \mathrm{mTorr}$ (Fig. $\left.4(\mathrm{~b})\right)$. Therefore, the grain size might play an important role in determining the $T_{C}$ of our films $33-35$.

\section{CONCLUSIONS}

In conclusion, we have successfully fabricated superconducting tantalum nitride thin films on magnesium oxide and oxidized silicon substrates using a pulsed laser ablation technique with infrared wavelength of $1064 \mathrm{~nm}$. By optimizing the growth parameters, we were able to obtain a maximum superconducting transition tempera- ture of $\sim 8$ and $6 \mathrm{~K}$ for films grown on $\mathrm{MgO}$ and $\mathrm{SiO}_{2}$, respectively. The surface morphology, crystal structure and electrical characteristics of the films were investigated. Films grown on $\mathrm{MgO}$ exhibited a face centered cubic phase, while an identical face centered cubic as well as a hexagonal phase coexisted in films grown on oxidized silicon. Both these phases are known to be mononitrides. We find that $\mathrm{MgO}$ is a better candidate for fabrication of high quality superconducting TaN thin films, as it offers a higher $T_{C}$ and lower resistivity, and is also more resistant to pressure fluctuation induced suppression of $T_{C}$.

\section{ACKNOWLEDGMENTS}

This research has been supported by Academy of Finland projects number 128532 and 260880. We thank Shen Boxuan for help with AFM measurements.
* sachaudh@jyu.fi

1 C. P. Poole, Handbook of superconductivity (Academic Press, San Diego, CA, 2000).

2 M. Wittmer, Applied Physics Letters 37, 540 (1980)

${ }^{3}$ K. Holloway, P. M. Fryer, J. Cyril Cabral, J. M. E. Harper, P. J. Bailey, and K. H. Kelleher, Journal of Applied Physics 71, 5433 (1992)

4 A. Katz, S. J. Pearton, S. Nakahara, F. A. Baiocchi, E. Lane, and J. Kovalchick, Journal of Applied Physics 73, 5208 (1993)

5 K. Baba and Hatada, Surface and Coatings Technology 84, 429 (1996).

6 A. Engel, A. Aeschbacher, K. Inderbitzin, A. Schilling, K. Il'in, M. Hofherr, M. Siegel, A. Semenov, and H.-W. Hübers, Applied Physics Letters 100, 062601 (2012).

7 A. B. Kaul, W. S. R., T. Van Duzer, L. Yu, N. Newman, and J. M. Rowell, Appl. Phys. Lett. 78, 99 (2001).

8 M. R. Nevala, I. J. Maasilta, K. Senapati, and R. C. Budhani, IEEE Trans. Appl. Supercond. 19, 253 (2009).

9 N. Terao, Japanese Journal of Applied Physics 10, 248 (1971)

10 H. Nie, S. Xu, S. Wang, L. You, Z. Yang, C. Ong, J. Li, and T. Liew, Applied Physics A 73, 229 (2001)

11 C. Stampfl and A. J. Freeman, Phys. Rev. B 71, 024111 (2005).

12 K. Reichelt, W. Nellen, and G. Mair, Journal of Applied Physics 49, 5284 (1978)

13 G. F. Hardy and J. K. Hulm, Phys. Rev. 93, 1004 (1954)

${ }^{14} \mathrm{~F}$. M. Kilbane and P. S. Habig, Journal of Vacuum Science and Technology 12, 107 (1975)

15 L. G. Boiko and S. V. Popova, Soviet Journal of Experimental and Theoretical Physics Letters 12, 70 (1970).

16 W. Ensinger, M. Kiuchi, and M. Satou, Journal of Applied Physics 77, 6630 (1995)

17 D. Gerstenberg and P. M. Hall, Journal of The Electrochemical Society 111, 936 (1964),

18 J. O. Olowolafe, C. J. Mogab, R. B. Gregory, and M. Kottke, Journal of Applied Physics 72, 4099 (1992)
19 C.-S. Shin, Y.-W. Kim, D. Gall, J. Greene, and I. Petrov, Thin Solid Films 402, 172 (2002).

20 S. M. Rossnagel, J. Vac. Sci. Technol. B 20, 2328 (2002),

${ }^{21}$ K. Il'in, M. Hofherr, D. Rall, M. Siegel, A. Semenov, A. Engel, K. Inderbitzin, A. Aeschbacher, and A. Schilling, Journal of Low Temperature Physics 167, 809 (2012)

22 A. Arranz and C. Palacio, Applied Physics A 81, 1405 (2005).

23 H. Kawasaki, K. D. J. Namba, and Y. Suda, MRS Proceedings 617, 10.1557/PROC-617-J3.22.

24 G. M. Matenoglou, L. E. Koutsokeras, C. E. Lekka, G. Abadias, S. Camelio, G. A. Evangelakis, C. Kosmidis, and P. Patsalas, Journal of Applied Physics 104, 124907 (2008).

25 S. Chaudhuri, M. Nevala, T. Hakkarainen, T. Niemi, and I. Maasilta, IEEE Trans. Appl. Supercond. 21, 143 (2011)

26 S. Chaudhuri, M. R. Nevala, and I. J. Maasilta, Submitted, arXiv:1301.6003

27 M. R. Nevala, S. Chaudhuri, J. Halkosaari, J. T. Karvonen, and I. J. Maasilta, Applied Physics Letters 101, 112601 (2012).

28 F. Giazotto, T. T. Heikkilä, A. Luukanen, A. M. Savin, and J. P. Pekola, Rev. Mod. Phys. 78, 217 (2006)

29 J. T. Muhonen, M. Meschke, and J. P. Pekola, Rep. Prog. Phys. 75, 046501 (2012).

${ }^{30}$ L. Li, N. Er-Wu, L. Guo-Hua, F. Wen-Ran, G. Wei-Chao, C. Guang-Liang, Z. Gu-Ling, F. Song-Hua, L. Chi-Zi, and Y. Si-Ze, Chinese Physics Letters 23, 3018 (2006).

31 N. P. Breznay, K. Michaeli, K. S. Tikhonov, A. M. Finkel'stein, M. Tendulkar, and A. Kapitulnik, Phys. Rev. B 86, 014514 (2012).

32 L. Kang, B. B. Jin, X. Y. Liu, X. Q. Jia, J. Chen, Z. M. Ji, W. W. Xu, P. H. Wu, S. B. Mi, A. Pimenov, Y. J. Wu, and B. G. Wang, Journal of Applied Physics 109, 033908 (2011). 
33 S. Matsuo, H. Sugiura, and S. Noguchi, Journal of Low Temperature Physics 15, 481 (1974).

34 K. Ohshima, T. Kuroishi, and T. Fujita, Journal of the Physical Society of Japan 41, 1234 (1976).
35 B. Abeles, R. W. Cohen, and G. W. Cullen, Phys. Rev. Lett. 17, 632 (1966). 